\title{
Educación Física en Chile: Una historia de la disciplina en los escritos de la primera publicación oficial del Instituto de Educación Física de la Universidad de Chile (1934-1962)
}

Physical Education in Chile: One Tells the History of the Discipline in the Writings of the First Official Publication of Physical Education Institute of the University of Chile

(1934-1962)

Educação Física em Chile: Uma história da disciplina nos escritos da primeira publicação oficial do Instituto de Educação Física da Universidade de Chile (1934-1962)

\author{
Carolina Poblete Gálvez, ${ }^{a}$ Alberto Moreno Doña, ${ }^{b}$ Enrique Rivera García ${ }^{c}$ \\ ${ }^{a}$ Universidad de Santiago de Chile. Fono: 56-02-27183538. \\ Correo electrónico: carolinapucv@gmail.com \\ b Pontificia Universidad Católica de Valparaíso, Chile. Fono: 56-032-2274383. \\ Correo electrónico: alberto.moreno@ucv.cl \\ c Universidad de Granada, España. Fono: 34-958-249641. \\ Correo electrónico: erivera@ugr.es
}

\begin{abstract}
RESUMEN
El trabajo que se presenta es resultado de una investigación mayor titulada La historia de la Educación Física en Chile a través de sus publicaciones académicas en la revista del Instituto de Educación Física de la Universidad de Chile. Esta publicación periódica de difusión del conocimiento científico, académico y técnico, dibuja el ideario educativo del siglo XX en temas del desarrollo corporal o motriz de la nación, cuando existía una sola universidad para la formación de estos profesionales. La investigación presentada a continuación muestra el resultado de las temáticas presentes en los artículos publicados entre su apertura en 1934 y hasta 1962, llegando a la conclusión de que los referentes intelectuales de la época contribuyeron al modelo que el Estado promovía en función del mejoramiento de la raza en términos biomédicos y la higiene moral como parte del ideario ciudadano.

Palabras clave: educación física, historia, publicación periódica.
\end{abstract}

\section{ABSTRACT}

The work presented is the result of a major research entitled The History of Physical Education in Chile through its Scholarly Publications in the Journal of Physical Education Institute of the University of Chile. This periodical publication of distribution of scientific, technical, and academic knowledge, draws the twentieth century development issues or motor body of the nation -when there was only one college for the training of these professionals. The research presented below shows the result of the themes included in the articles published between its opening in 1934 until 1962, concluding that the intellectuals of the time contributed to promoting the state model based on improving the race in biomedical terms and moral hygiene as part of the citizens' ideology.

Key words: physical education, history, periodical publication.

\section{RESUMO}

Resultado de uma grande pesquisa intitulada A História da Educação Física no Chile, a partir das publicações acadêmicas no Jornal do Instituto de Educação Física da Universidade do Chile, mostra o resultado dos temas 
presentes nos artigos publicados entre 1934 até 1962. Esta publicação periódica, de divulgação do conhecimento científico e acadêmico, da ideologia técnico-educacional, desenha as questões do desenvolvimento do século XX ou no corpo do motor da nação, quando havia apenas uma faculdade para a formação desses profissionais. Conclui-se que os intelectuais da época contribuiram para promover o modelo de Estado baseado na melhoria da raça em termos biomédicos e de higiene moral, como parte da deologia cidadã.

Palavras chave: educação física, história, publicação periódica.

\section{INTRODUCCIÓN}

El Instituto de Educación Física y Manual $^{1}$ de la Universidad de Chile, entidad del Estado para la formación de profesionales de la Educación Física (en adelante EF), comienza su labor en 1906 y es considerado como la primera institución formadora de profesionales exclusiva de la disciplina en Latinoamérica (Instituto de EF, 1936; Gutiérrez, 1933; Salas, 2009). Los datos históricos señalan que fue, durante 58 años, el único organismo que preparó a profesionales especialistas para la enseñanza de la EF en Chile. Transcurridos 28 años desde su instauración, el instituto publica, en 1934, el primer Boletín de $\mathrm{EF}^{2}$, declarándose como parte representativa del organismo oficial de la casa de estudios. El objetivo central de esta publicación se explicita en su primer número.

El gran progreso experimentado por las Ciencias Biológicas y los avances que ha hecho la Pedagogía Científica en los últimos años, han repercutido lógicamente en las orientaciones de la EF y han planteado nuevos e interesantes problemas a quienes con ella se ocupan. Este boletín tratará de tener a sus lectores al corriente de las novedades que en los principales centros científicos y culturales se vayan produciendo (Instituto de EF, 1934: s/p).

La responsabilidad autoimpuesta por este organismo revela varias interrogantes en relación con la forma de validación del conocimiento profesional de la enseñanza, y de la utilidad de las clases de EF en diferentes contextos: la escuela, la academia, el deporte de masas, las políticas estatales de la época y los grandes referentes internacionales. ¿Cuál es el valor histórico contextual que la Revista Chilena Educación de Física puede entregar como registro de la génesis y evolución de la disciplina en el país? Considerando que esta publicación se crea específicamente para la difusión del conocimiento en el área, ¿cuáles son las temáticas que fueron centro de atención para el desarrollo del conocimiento y validación de la EF en el segundo tercio del siglo XX?

Los estudios de la historia de la Educación Física en Chile son muy escasos. Sólo existen algunos escritos acerca de temas más específicos como la historia del deporte (Muñoz, 2001), algunas aproximaciones a la historia desde lo educativo que aportan datos desde los fines de la EF en sus orígenes institucionales (Martínez, 2012), y la definición de los diferentes momentos vividos por la EF en relación a su desarrollo y fines (Cornejo, Matus y Vargas, 2011), aunque con limitado contraste historiográfico. En este sentido, el objetivo de esta investigación es analizar y comprender contextualizadamente los datos históricos y temáticos que la Revista Chilena Educación de Física podría aportar desde su génesis en 1934 y hasta 1962.

La denominación de Manual sólo se utilizó en su primera década, luego cambió a Instituto de EF, y posterior a 1950 a Instituto de EF y Técnica.

2 A partir de 1950 cambiaría su nombre a Revista Chilena de EF. 


\section{CONTEXTO DE LA INVESTIGACIÓN}

El sistema educacional chileno se gestó, durante el siglo XX, bajo las bases de un estado docente que se haría cargo de todo aquello relacionado con la educación, proceso que tuvo pleno protagonismo hasta 1973. La educación chilena evoluciona, a partir del siglo XIX, desde el conservadurismo autoritario, pasando por el liberalismo laicista, hasta el realismo positivista del siglo XX, y el pragmatismo educativo actual (Flores y Rivera, 2003). Letelier, en 1892 (Cit. en Ruiz, 2010), comenta que la agencia educacional era el Estado, y la universidad era la encargada de conducir la política modernizadora. Las declaraciones de los intelectuales de la época reconocen, en este sentido, el papel de la universidad y su relación con las políticas de gobierno. La historia de la educación chilena del siglo XX responde a expectativas desarrollistas y nacionalistas fundadas en las ciencias positivistas (Godoy, 2007; Hevia, Fernández y Home, 2010; Labarca, 1939; Ruiz, 2010). La formación universitaria de los profesores secundarios es un elemento central de la política. A través de la mediación de los profesores, la cohesión social desciende entonces desde la cima de la institución escolar, desde la acción de las universidades, hacia la base social (Ruiz, 2010).

El fundamento de la educación chilena estaba centrado en una educación para la nación y su desarrollo económico, construido a partir de un currículum de utilidad práctica para la enseñanza, y operacionalizado a través de instituciones orientadas y fiscalizadas desde el Estado, como fue el caso del Instituto de EF de la Universidad de Chile. "La nueva escuela tendería a desarrollar aptitudes que fuesen aprovechadas en el plan de industrialización que se estaba implementando. Dentro de este esquema la Universidad de Chile asumió un rol como centro más importante de formación profesional" (Moulian, 2006: 654). Todo ello se fundamentó en las eventuales necesidades de los sectores más pobres de la población (salubridad, higiene y mejoramiento de la raza), los cuales se convirtieron en la piedra de tope de la instalación del ideario capitalista que la clase política quería erigir como alternativa de progreso. En otras palabras, la raza chilena debía adaptarse a la lógica de progreso que el Estado había asumido. Al decir de Galdames,

La educación económica de las clases populares, especialmente podría contribuir a revertir la negativa situación social y étnica del país y contribuir a transformarnos en un pueblo manufacturero mercantil i marino, lo que es un imperativo para la época, regida por la Lei científica de la lucha selectiva (1912: 135-137).

Esta perspectiva ha estado presente durante todo el siglo XX, dando sustento a las teorías desarrollistas y de mercado en el contexto educativo (Ruiz, 2010). Sin duda que ha evolucionado, pero en ningún caso ha desaparecido, pues nos ha llevado al sistema educativo de mercado que actualmente tenemos. En este sentido, la búsqueda de la historia y las consecuencias de los modelos educativos promovidos por instituciones educativas como la Universidad de Chile, aportan una importante arista de análisis para muchas de las validaciones del sistema educativo actual.

Si consideramos que Chile, en lo que a políticas públicas se refiere, ha evolucionado desde una idea de educación como medio de formación ciudadana para la república, para pasar a un enfoque orientado al desarrollo y la modernización, y de ahí culminar en la actual orientación de mercado, no es menos relevante preguntarse: ¿cuál es el rol 
educativo de la EF para un país que busca desarrollo económico? Parte de la respuesta a esta pregunta puede encontrarse en aquellos organismos que regularon y dictaron las bases de su desarrollo como disciplina. Nos referimos, particularmente, a la publicación del Instituto de EF de la Universidad de Chile (1934-1962), la que en 28 años muestra una historia no contada de la EF en Chile. Esta publicación que se declara, explícitamente, como órgano representante de la universidad, presenta diferentes tipos de contribuciones, a saber: discusiones, publicaciones científicas, ensayos, propuestas metodológicas y crónicas contingentes para la sociedad chilena.

Nuestro interés se centra en conocer la historia que subyace a la propia identidad de la disciplina, y cómo ésta está ligada al cuestionamiento de su propia existencia. Esto atañe, por consiguiente, a la forma en que se ha enseñado, bien comprendiendo el conocimiento como histórico, contextualizado, dinámico, o en forma más reproductiva o mecanicista, eliminando la incertidumbre y buscando respuestas estandarizadas (Toro, 2005). Nos preguntamos, entonces: $¿$ ha trascendido, evolucionado o se ha perpetuado el ideario de la EF en la historia reciente de Chile?

El conocimiento que un país tiene acerca de su historia le hace caracterizarse por ciertos patrones que lo diferencian de los otros, los hechos escritos y las creaciones de quienes la escriben proyectan las motivaciones de su propia imagen, determinando el sentido de esa historia contada (Aurell, 2008). Por esta razón, es importante conocer no sólo el hecho de la historia, sino también la contingencia en la que se escribe y la intencionalidad de quienes la construyen.

Pensamos que la publicación objeto de esta investigación presenta las características idóneas para rastrear el sentido y la identidad del área de EF en el periodo analizado, ya que en el Chile de principios del siglo XX, sin importar su temática o su carácter, las publicaciones universitarias se constituían en motores de creación y difusión del pensamiento (Vásquez y Vásquez, 2011).

\section{ANTECEDENTES HISTÓRICOS DE LA EDUCACIÓN FÍSICA EN CHILE}

Los antecedentes que existen en relación a la historia de la EF en Chile, además de escasos, son un claro ejemplo de cómo el proceso de aprendizaje y el rol social de la disciplina se han asociado, casi exclusivamente, al ámbito deportivo (Marón, 2007; Modiano, 1997; Muñoz, 2001), estableciéndose una relación directa y acrítica entre deporte y educación. Al buscar, minuciosamente, los referentes de la práctica educativa-física desde una esfera pedagógica-escolar es posible identificar la disputa de dos grandes perspectivas: la primera representada por la gimnasia alemana, la segunda por la gimnasia sueca. Las ideas de ambas corrientes gimnásticas se instalaron como foco de análisis de los intelectuales de la época en relación a la disciplina, estableciéndose una clara disputa entre los que defendían una de las dos corrientes mencionadas y rechazando la otra. "Aquí en Chile, siempre he creído que para abrir una polémica razonada acerca de ambos sistemas de EF no debemos personificar los hechos y atacar. (...) ¿Por qué pues, nosotros no imitamos el ejemplo que dan estas naciones y formamos también nuestro sistema de EF?" (Matus, 1904: 34-52).

A partir de este punto se abren otras posibilidades reflexivas, como las planteadas en el Congreso Pedagógico de 1889. En él se declaraba el necesario carácter obligatorio de la EF en las escuelas chilenas y la organización del primer curso para profesores de gimnasia. 
Las características de la enseñanza de la EF hacia finales del siglo XIX y principios del siglo XX estuvieron centradas en la higiene y la moral, siendo coherente con las dos perspectivas presentes pero, además, con el modelo de validación del conocimiento altamente calificado desde el pensamiento europeo: el positivismo. Valentín Letelier, intelectual chileno destacado en el ámbito educativo y afín con el pensamiento positivista de la educación, señala que "en el sistema integral de educación positiva: la higiene, la jimnástica (...) enseñanza que propende a desarrollar los órganos físicos" (Cit. en Flores y Riviera, 2003: 156) deben ser incluidas. Nos encontramos, entonces, con una EF instrumental que busca, a través de la instrucción, la obtención de beneficios para una sociedad productiva (Martínez, 2012). Existió, también, una EF para las masas populares cuyos propósitos principales eran el fortalecimiento de la raza, el desarrollo del trabajo industrial y la consecución de una fuerte disciplina corporal, y una EF para la clase dirigente focalizada en la satisfacción de sus impulsos y necesidades físicas para la competencia, el ocio y los deportes.

El deporte mundializado llega a Chile a través de los ingleses y otros inmigrantes. Ello fue consecuencia de una fuerte inversión privada a través de clubes deportivos constituidos jurídicamente y validados por la sociedad en su conjunto, con el fin de recrear a la clase más acomodada y para formar hábitos en la clase obrera, que por esa época sufría las consecuencias de la migración del campo a la cuidad (hacinamiento, enfermedades, analfabetismo citadino, entre otras). La introducción del deporte en la escuela fue progresivamente formando parte del eje vertebrador de la EF, aunque existieron detractores por la excesiva competitividad que representaban quienes la promovían, en función de las ciencias médicas, otros tipos de ejercicios más ligados a la salud individual y colectiva (Martínez, 2012).

Salud y deporte influenciaron el desarrollo y el progreso de la nación bajo el modelo educativo nacionalista y desarrollista chileno. Los lineamientos educativos bajo este modelo consideraban la relevancia tanto de los 'cuerpos' saludables, como de los 'cuerpos' productivos para el país. Por ello, el sentido de "la enseñanza de la EF cobró importancia como signo de una transformación morfológica promotora del bienestar sanitario de la población y del crecimiento de la nación, permitiendo a su vez integrarse como un saber escolar eminentemente moderno, fundamental para la educación integral" (Martínez, 2012: 1).

En resumen, el contexto histórico de la EF chilena en el primer tercio del siglo XX muestra el sentido de la disciplina, su orientación a la salud de la población, en términos utilitarios, buscando una mayor producción económica. Copiando la perspectiva positivista de modelos extranjeros, se potencia el concepto de "raza" como determinante para el desarrollo nacional. Frente a estos antecedentes nos surgen algunas preguntas que han orientado nuestro trabajo investigativo. Considerando la Revista Chilena de EF como ideario de la disciplina durante los años 1934-1962, ¿cuáles fueron las grandes temáticas y problemáticas abordadas por los intelectuales de la época en la publicación mencionada?, y ¿cómo estas temáticas evolucionaron en el transcurso de las cuatro décadas analizadas?

\section{METODOLOGÍA}

La forma de registrar la historia y su utilidad siguen siendo un aporte al conocimiento científico. Pero el sentido que se le dé a los registros históricos dependerá, en gran parte, del valor, en términos de significación, del objeto de estudio. Los distintos tipos de documentos y fuentes de información que sirven para una investigación histórica pueden 
ser tan variados como formas existan de interpretarla. Sin embargo, reconocer que el pasado queda impreso en la creación humana, a través del lenguaje, requiere de un especial tratamiento que permita ponerlo al servicio del presente.

Entendiendo el potencial histórico de la publicación periódica del Instituto de EF de la Universidad de Chile, entre los años 1934 y 1962, en nuestra investigación se ha buscado abrir caminos de comprensión sobre las temáticas que toman relevancia presencial histórica en la producción intelectual de los temas educativo-físicos, con el fin de verificar si las temáticas de debate actuales vienen acompañadas de esta carga histórica. Se abordan los datos históricos, bajo el modelo de investigación cuantitativa, descriptiva y transaccional, identificando las temáticas abordadas por los intelectuales nacionales y extranjeros que en dicha publicación escribieron y analizaron la disciplina en las cuatro décadas investigadas. Los artículos analizados corresponden a un total de 842, entre los cuales se encuentran ensayos, artículos originales y experiencias pedagógicas.

El análisis cuantitativo acometido se centra en las temáticas presentes en los títulos de los artículos. Para la clasificación y categorización de las temáticas escogimos como referente el Tesauro de la base de datos francesa "Heracles", referente europeo en el ámbito de la información y documentación de la disciplina en estudio, y lugar donde se encuentra el servicio de información y documentación SportDoc, eje central de la base de datos (Remans, 2000).

El primer paso a seguir en nuestro procedimiento metodológico consistió en agrupar y categorizar inductivamente los artículos en función de las seis grandes categorías del Tesauro mencionado, tal como se observa en la Tabla 1.

Tabla 1. Orientaciones para la clasificación de artículos analizados

\begin{tabular}{|l|l|}
\hline \multicolumn{1}{|c|}{ Orientaciones } & \multicolumn{1}{c|}{ Descripción } \\
\hline Cuerpo biológico & $\begin{array}{l}\text { Títulos que presentaban conceptos asociados a la explicación del cuerpo } \\
\text { humano en términos científicos biologicistas. }\end{array}$ \\
\hline Formación & $\begin{array}{l}\text { Títulos que contenían conceptos relacionados con la educación profesional, } \\
\text { universitaria y escolar. }\end{array}$ \\
\hline Organización & $\begin{array}{l}\text { Títulos que mostraban como foco neurálgico del trabajo la descripción y/o } \\
\text { análisis de organizaciones institucionales o de agrupaciones de diversa índole. }\end{array}$ \\
\hline Motricidad & $\begin{array}{l}\text { Títulos en donde las actividades motrices eran el eje vertebrador: deportes, } \\
\text { gimnasia, expresión, recreación y ludicidad. }\end{array}$ \\
\hline Humanidades & $\begin{array}{l}\text { Títulos en donde se explicitaban conceptos asociados a las ciencias sociales en } \\
\text { general: sociología, historia, psicología y geografía, referenciando } \\
\text { descripciones particulares de algún hecho. }\end{array}$ \\
\hline Personajes & $\begin{array}{l}\text { Títulos en donde se hacía especial mención a alguna persona destacada en la } \\
\text { EF y/o a la labor profesional de ella. }\end{array}$ \\
\hline
\end{tabular}

Fuente: elaboración propia.

Una vez realizada esta primera categorización en base al título del artículo y su contenido, se procedió a un segundo y tercer nivel de indización (Tabla 2), siguiendo 
las subcategorías propuestas en el Tesauro de la base de datos "Heracles" (18 grandes categorías, 46 subcategorías en primer nivel y 20 subcategorías en segundo nivel). Es importante mencionar que si en la categorización del primer nivel se mantuvo el criterio de pertenencia exclusiva a una sola categoría, en los otros dos niveles se permite la inclusión de un artículo en dos o más subcategorías.

Tabla 2. Categorías organizadas en base a la orientación creada para la clasificación Tesauro francés

\begin{tabular}{|l|l|}
\hline \multicolumn{1}{|c|}{ Orientaciones temáticas } & \multicolumn{1}{c|}{ Categorías temáticas Tesauro francés } \\
\hline Cuerpo biológico & $\begin{array}{l}\text { Fisiología, anatomía, biometría, análisis del movimiento, sexo } \\
\text { (femenino-masculino), ciencias médicas y ciencias básicas. }\end{array}$ \\
\hline Formación & Enseñanza, educación, aprendizaje y metodologías técnicas. \\
\hline Organización & Institución, política y competición. \\
\hline Motricidad & Actividad física. \\
\hline Humanidades & Ciencias humanas y geografía. \\
\hline Personajes & Oficios y funciones. \\
\hline
\end{tabular}

Fuente: elaboración propia.

A partir de esta categorización nos surgieron nuevas preguntas de investigación: ¿a qué cantidad de escritos corresponde cada temática?; ¿cómo se distribuye cada categoría a lo largo de los 28 años de la publicación seriada?; ¿cuál es la relación entre la distribución de las temáticas a lo largo de los años y la contingencia sociohistórica de la educación chilena en esa época?, y; ¿qué puede decirse de las categorías identificadas en relación con la historia de la EF chilena?

Para tratar de dar respuesta a las cuestiones anteriores, se realizó un estudio descriptivo y correlacional utilizando el software de análisis estadístico SPSS.19. Concretamente se utilizó un análisis comparativo de frecuencias en base a las décadas que involucra el periodo estudiado en la revista, la aplicación de la prueba estadística de independencia Chicuadrado, y para poder profundizar en la relación entre las categorías (variable dependiente) respecto a las décadas en las que aparecen (variable independiente), se procedió a un análisis estadístico bivariante. Debemos tener en cuenta que el nivel de significación es del 5\%.

\section{RESULTADOS Y DISCUSIÓN}

En este apartado evidenciamos la presencia cuantitativa de las temáticas. La expresamos en términos de frecuencia-porcentaje y relación bivalente entre las categorías del estudio. Mostramos también la relación de lo obtenido con el contexto histórico, político y educacional de la época, todo ello separado por décadas, pues creemos que ayuda a clarificar los hallazgos alcanzados. 


\subsection{PORCENTAJE DE FRECUENCIA PRESENCIAL DE LAS TEMÁTICAS CLASIFICADAS}

Uno de los primeros resultados de esta investigación (ver Tabla 3) corresponde a la cantidad de escritos por cada orientación temática y su relación porcentual en base al total de escritos.

Tabla 3. Cantidad ${ }^{3}$ y porcentaje de escritos categorizados según orientación temática

\begin{tabular}{|l|c|c|}
\hline $\begin{array}{c}\text { Orientación para la elección de } \\
\text { categorías Tesauro francés }\end{array}$ & $\begin{array}{c}\text { Cantidad de } \\
\text { escritos }\end{array}$ & $\begin{array}{c}\text { Porcentaje con relación al total } \\
\text { de escritos (842) }\end{array}$ \\
\hline Cuerpo Biológico & 355 & $42 \%$ \\
\hline Formación & 260 & $31 \%$ \\
\hline Organización & 90 & $11 \%$ \\
\hline Motricidad & 374 & $44 \%$ \\
\hline Humanidades & 339 & $40 \%$ \\
\hline Personajes & 92 & $11 \%$ \\
\hline
\end{tabular}

Fuente: elaboración propia.

Es evidente la alta cantidad de escritos relacionados con la orientación motricidad (44\%), en cuanto a los tipos de prácticas asociadas a la EF y el deporte, una lógica bastante esperada si consideramos el tipo de revista especializada que constituye el cuerpo de nuestro análisis. Sin embargo, la segunda orientación con mayor presencia corresponde a la perspectiva biológica desde la que se analiza el cuerpo y lo corporal (42\%), mostrando una clara orientación científica de la disciplina bajo un enfoque biomédico. Es relevante considerar que son siete las categorías que están relacionadas con esta orientación, por lo que no hay duda de que son las ciencias exactas y médicas las que sustentan la mayor parte de las publicaciones de la época. Las humanidades (40\%) concentran mayor número de artículos en la categoría geografía. Esto nos muestra la importancia de rescatar las experiencias particulares, tanto nacionales como internacionales, nacidas en diferentes contextos.

En un nivel con menor presencia porcentual aparecen aquellas orientaciones relacionadas con la formación, como la enseñanza, los tipos de educación (universitaria y/o escolar), el aprendizaje y ciertas terminologías técnicas. Posteriormente, se evidencia el conjunto de escritos relacionados con el reconocimiento de algunos personajes destacados y/o su función como actores sociales. Por último, se muestran escritos con diversos antecedentes organizativos. Si bien las temáticas orientadas a motricidad y cuerpo biológico son las que mayor presencia tienen, es importante considerar que la primera sólo

Varios de los escritos categorizados poseen la particularidad de pertenecer a más de una categoría, característica que se decidió respetar al momento de la categorización. Es por esta razón que el total de escritos no es colocado al final de la tabla pues ésta sobrepasaría los 842 escritos realmente existentes. Los escritos categorizados en una sola categoría corresponden a 269, mientras que los que son categorizables en más de una corresponden a 573. 
tiene una categoría (actividad física), mientras que la segunda posee siete. Esto tiene como consecuencia que los escritos categorizados a partir de la orientación biológica poseen una mayor variabilidad al representarlos en su presencia temática.

Para graficar más fielmente esta última idea, se consideran las 18 categorías en forma independiente (Tablas 4a, 4b, 4c y 4d), mostrando los resultados de los porcentajes de artículos por: "Categoría", "1a subcategoría" y " $2^{a}$ subcategoría". La clasificación "Sî" corresponde al número de escritos en el que aparece esta temática; "No" corresponde al número de escritos que no contienen la temática.

Para cada subcategoría, el número de datos sobre el que se estudia está restringido a los casos de su categoría superior en la que se ha dado esa temática. Es decir, si hemos observado que un escrito está contenido en una temática en particular, entonces: ¿en cuántos de ellos se observa la subcategoría de la temática en sí? Esto se responde porcentualmente pero también numéricamente entre paréntesis.

Tabla $4 a$. Porcentaje de escritos (frecuencia) por temática, $1^{\mathrm{a}}$ subcategoría y $2^{\mathrm{a}}$ subcategoría de la temática

\begin{tabular}{|l|l|c|c|}
\hline \multicolumn{2}{|l|}{} & Sí & No \\
\hline \multirow{2}{*}{ 1. Fisiología } & 1.1. Fisiología del ejercicio & $13,7 \%(115)$ & $86,3 \%(727)$ \\
\cline { 2 - 4 } & 1.2. Funciones fisiológicas & $72,2 \%(83)$ & $27,8 \%(32)$ \\
\cline { 2 - 4 } & 1.3. Edad biológica & $19,1 \%(22)$ & $80,9 \%(93)$ \\
\hline \multirow{2}{*}{ 2. Anatomía } & 2.1. Características antropométricas & $8,7 \%(10)$ & $91,3 \%(105)$ \\
\cline { 2 - 5 } & 2.2. Patologías anatómicas & $2,6 \%(22)$ & $97,4 \%(820)$ \\
\hline \multirow{2}{*}{ 3. Biometría } & & $27,3 \%(6)$ & $72,7 \%(16)$ \\
\hline 4. Análisis del movimiento & $1,7 \%(14)$ & $98,3 \%(828)$ \\
\hline 5. Sexo femenino-masculino & $1,4 \%(12)$ & $98,6 \%(830)$ \\
\hline 6. Ciencias Médicas & $4,4 \%(37)$ & $95,6 \%(805)$ \\
\hline \multirow{5}{*}{} & 6.1. Medicina deportiva & $17,3 \%(146)$ & $82,7 \%(696)$ \\
\cline { 2 - 5 } & 6.2. Rehabilitación-terapia & $23,3 \%(34)$ & $76,7 \%(112)$ \\
\cline { 2 - 5 } & 6.3. Alimentación-nutrición & $15,1 \%(22)$ & $84,9 \%(124)$ \\
\cline { 2 - 5 } & 6.4. Salud & $31,5 \%(46)$ & $68,5 \%(100)$ \\
\cline { 2 - 5 } & 6.5. Accidentes traumáticos & $4,1 \%(6)$ & $95,9 \%(140)$ \\
\hline 7. Ciencias Exactas & $1,1 \%(9)$ & $98,9 \%(833)$ \\
\hline 8. Enseñanza & & $7,4 \%(62)$ & $92,6 \%(780)$ \\
\hline
\end{tabular}

Fuente: elaboración propia. 
Estudios Pedagógicos XL, $\mathrm{N}^{\circ}$ 2: 265-282, 2014

EDUCACIÓN FÍSICA EN CHILE: UNA HISTORIA DE LA DISCIPLINA EN LOS ESCRITOS DE LA PRIMERA

PUBLICACIÓN OFICIAL DEL INSTITUTO DE EDUCACIÓN FÍSICA DE LA UNIVERSIDAD DE CHILE (1934-1962)

En la Tabla 4a podemos ver la fuerte presencia de escritos relacionados con la fisiología del ejercicio y las características antropométricas, en torno al $72 \%$ ambas subcategorías. Por el contrario, se evidencia la escasa presencia de otras temáticas como la edad biológica y los accidentes traumáticos (por debajo del 10\%), seguidos por las funciones fisiológicas, patologías anatómicas, medicina deportiva, rehabilitación y alimentación (por debajo todas ellas del 30\% de presencia en la revista).

Si cambiamos la mirada a las temáticas relacionadas directamente con la EF, podemos observar en la Tabla $4 \mathrm{~b}$ una presencia muy discreta de escritos en esta dirección. Las que mayor presencia tienen son las relacionadas con los estudiantes, que es la gran excepción con un $75 \%$, seguida de la pedagogía, los métodos de EF, la didáctica, la enseñanza superior y la escuela que se sitúan en unos valores entre el $30 \%$ y el $45 \%$. En la parte baja destacan las temáticas relacionadas con materiales y recursos, los actores y los programas de estudios, que se sitúan con una presencia por debajo del $10 \%$.

Tabla $4 b$. Porcentaje de escritos (frecuencia) por temática, $1^{a}$ subcategoría y $2^{a}$ subcategoría de la temática

\begin{tabular}{|c|c|c|c|}
\hline & & Sí & No \\
\hline \multicolumn{2}{|c|}{ 8. Enseñanza } & $7,4 \%(62)$ & $92,6 \%(780)$ \\
\hline & 8.1. Pedagogía & $30,6 \%(19)$ & $69,4 \%(43)$ \\
\hline & 8.2. Métodos de EF & $38,7 \%(24)$ & $61,3 \%(38)$ \\
\hline & 8.3. Didáctica & $30,6 \%(19)$ & $69,4 \%(43)$ \\
\hline \multicolumn{2}{|c|}{ 9. Educación } & $21,1 \%(178)$ & $78,9 \%(664)$ \\
\hline & 9.1. Formación profesional & $11,8 \%(21)$ & $88,2 \%(157)$ \\
\hline & 9.2. Formación continua & $29,8 \%(53)$ & $70,2 \%(125)$ \\
\hline & 9.3. Sistema educativo & $30,9 \%(55)$ & $69,1 \%(123)$ \\
\hline & 9.3.1. Enseñanza superior-universidad & $45,5 \%(25)$ & $54,5 \%(30)$ \\
\hline & 9.3.2. Escuela & $32,7 \%(18)$ & $67,3 \%(37)$ \\
\hline & 9.3.3. Enseñanza secundaria & $21,8 \%(12)$ & $78,2 \%(43)$ \\
\hline \multicolumn{2}{|r|}{ 9.4. Materiales y recursos } & $4,5 \%(8)$ & $95,5 \%(170)$ \\
\hline & 9.5. Actores escolares & $4,5 \%(8)$ & $95,5 \%(170)$ \\
\hline & 9.5.1. Estudiante universitario & $25,0 \%(2)$ & $75,0 \%(6)$ \\
\hline & 9.5.2. Estudiante & $75,0 \%(6)$ & $25,0 \%(2)$ \\
\hline & 9.6. Orientación escolar & $11,2 \%(20)$ & $88,8 \%(158)$ \\
\hline & 9.7. Programas de estudios & $7,3 \%$ & $92,7 \%(165)$ \\
\hline \multicolumn{2}{|c|}{ 10. Aprendizaje } & $0,6 \%(5)$ & $99,4 \%(837)$ \\
\hline \multicolumn{2}{|c|}{ 11. Metodologías técnicas } & $1,8 \%(15)$ & $98,2 \%(827)$ \\
\hline
\end{tabular}

Fuente: elaboración propia. 
Estudios Pedagógicos XL, $\mathrm{N}^{\circ}$ 2: 265-282, 2014

EDUCACIÓN FÍSICA EN CHILE: UNA HISTORIA DE LA DISCIPLINA EN LOS ESCRITOS DE LA PRIMERA PUBLICACIÓN OFICIAL DEL INSTITUTO DE EDUCACIÓN FÍSICA DE LA UNIVERSIDAD DE CHILE (1934-1962)

En la Tabla 4c destacan con claridad los temas relacionados con el Estado (90\%), que despiertan un alto interés generando gran número de escritos sobre la temática. Lejos de ella, aparecen las competencias internacionales con una presencia del 64,5\%.

Tabla $4 c$. Porcentaje de escritos (frecuencia) por temática, $1^{\text {a }}$ subcategoría y $2^{\text {a }}$ subcategoría de la temática

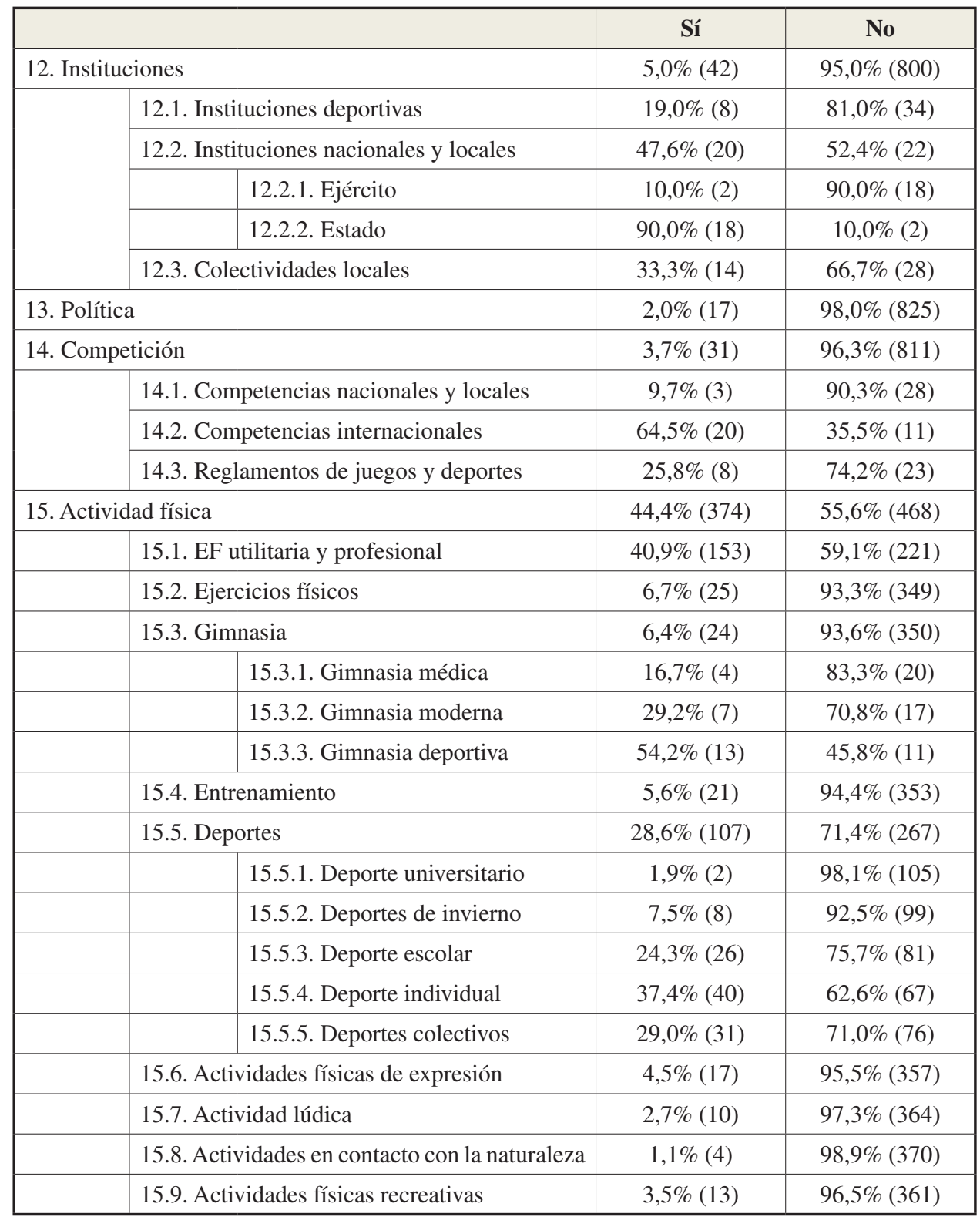

Fuente: elaboración propia. 
Estudios Pedagógicos XL, $\mathrm{N}^{\circ}$ 2: 265-282, 2014

EDUCACIÓN FÍSICA EN CHILE: UNA HISTORIA DE LA DISCIPLINA EN LOS ESCRITOS DE LA PRIMERA

PUBLICACIÓN OFICIAL DEL INSTITUTO DE EDUCACIÓN FÍSICA DE LA UNIVERSIDAD DE CHILE (1934-1962)

Por el contrario, llama la atención que una revista de EF presente unos índices tan bajos de presencia en temáticas relacionadas con la actividad física. Dentro de ella, las subcategorías de ejercicios físicos, gimnasia, entrenamiento, deporte universitario, deportes de invierno, actividades físicas de expresión, actividades lúdicas que se desarrollan en entornos naturales y recreativas, obtienen unos valores por debajo del $10 \%$.

Tabla $4 d$. Porcentaje de escritos (frecuencia) por temática, $1^{\mathrm{a}}$ subcategoría y $2^{\mathrm{a}}$ subcategoría de la temática

\begin{tabular}{|c|c|c|c|}
\hline & & Sí & No \\
\hline \multicolumn{2}{|c|}{ 16. Ciencias-Humanidades } & $17,7 \%(149)$ & $82,3 \%(693)$ \\
\hline & 16.1. Sociología & $46,7 \%(70)$ & $53,3 \%(79)$ \\
\hline & 16.1.1. Cultura & $50,0 \%(35)$ & $50,0 \%(35)$ \\
\hline & 16.1.2. Práctica deportiva & $22,9 \%(16)$ & $77,1 \%(54)$ \\
\hline & 16.1.3. Educación popular & $27,1 \%(19)$ & $72,9 \%(51)$ \\
\hline & 16.2. Filosofía-Estética & $5,3 \%(8)$ & $94,7 \%(141)$ \\
\hline & 16.3. Historia & $33,3 \%(50)$ & $66,7 \%$ (99) \\
\hline & 16.4. Categoría de edad & $4,0 \%(6)$ & $96,0 \%$ (143) \\
\hline & 16.4.1. Infancia & $66,7 \%(4)$ & $33,3 \%(2)$ \\
\hline & 16.4.2. Edad-Adolescente & $33,3 \%(2)$ & $66,7 \%(4)$ \\
\hline & 16.5. Psicología & $10,7 \%(16)$ & $89,3 \%(133)$ \\
\hline \multicolumn{2}{|l|}{ 17. Geografía } & $22,4 \%(189)$ & $77,6 \%(653)$ \\
\hline & 17.1. Europa & $46,0 \%(87)$ & $54,0 \%(102)$ \\
\hline & 17.2. América Latina & $52,9 \%(100)$ & $47,1 \%(89)$ \\
\hline & 17.3. Otros & $1,1 \%(2)$ & $98,9 \%(187)$ \\
\hline \multicolumn{2}{|c|}{ 18. Oficios y funciones } & $10,9 \%(92)$ & $89,1 \%(750)$ \\
\hline & 18.1. Educador & $66,3 \%(61)$ & $33,7 \%(31)$ \\
\hline & 18.2. Profesionalismo & $28,3 \%(26)$ & $71,7 \%(66)$ \\
\hline & 18.3. LING-PER-HENRIK & $5,4 \%(5)$ & $94,6 \%$ (87) \\
\hline
\end{tabular}

Fuente: elaboración propia.

Por último, podemos observar en la Tabla 4 d cómo hay un número significativo de subcategorías que se sitúan por encima del $45 \%$ de presencia, asunto que es digno de considerar si se tiene en cuenta que son temáticas complementarias. Podemos citar entre ellas: la sociología, la cultura, los temas en relación a la infancia, los escritos localizados en Europa y América Latina, y la figura del educador. En la otra cara, las temáticas con escasa presencia se concentran en los temas de filosofía y psicología que tienen una presencia de un $5,3 \%$ y un $10,9 \%$, respectivamente. 
EDUCACIÓN FÍSICA EN CHILE: UNA HISTORIA DE LA DISCIPLINA EN LOS ESCRITOS DE LA PRIMERA PUBLICACIÓN OFICIAL DEL INSTITUTO DE EDUCACIÓN FÍSICA DE LA UNIVERSIDAD DE CHILE (1934-1962)

\subsection{ANÁLISIS BIVALENTE ENTRE TEMÁTICAS Y DÉCADAS DE PUBLICACIÓN}

El total de años analizados para esta publicación se agrupan en cuatro décadas:

- Primera (1930-1939): con 163 escritos $(19,4 \%)$

- Segunda (1940-1949): con 258 escritos $(30,6 \%)$

- Tercera (1950-1959): con 341 escritos $(40,5 \%)$

- Cuarta (1960-1969): con 80 escritos (9,5\%)

Debemos tener en cuenta que sólo hemos considerado las 18 categorías ya presentadas como variables dependientes, sin considerar las subcategorías que contienen.

Como podemos ver en la Gráfica 1, respecto a la temática "Fisiología", en la primera década se publicaron más escritos que en las otras tres. En la temática "Anatomía" no se publicó nada en la última década y durante la primera se publicaron más que en las dos siguientes. El número de escritos para esta temática, según las décadas, fueron significativos con un p-valor de 0.011. "Biometría" aparece en un $2 \%$ de los escritos durante la primera y la tercera década, desapareciendo prácticamente en la segunda y cuarta. Por su parte, "Análisis del movimiento" apareció en el 1\% de los escritos de cada década. En particular, en la tercera década, se publicaron 5 escritos de 341 y en la segunda fueron 4 de 258 . Para la temática "Sexo", en la última década se escribieron 5 de los 80 publicados para esos años. La década en la que más se publica sobre sexo es la segunda, con 13 de los 258 publicados. Las publicaciones sobre "Ciencias médicas" se concentran especialmente en la tercera y segunda década, con un $19 \%$ y un $18 \%$, respectivamente. Por último, de los nueve escritos (1,7\% del total) que se publicaron durante las cuatro décadas para "Ciencias exactas" 6 pertenecen a la tercera década.

Sintetizando este primer bloque de análisis de las categorías con una clara orientación a un enfoque de "Cuerpo biológico", es posible notar una presencia constante de estas temáticas a lo largo de las cuatro décadas, con la sola excepción de los temas relacionados con anatomía y biometría, de los cuales no hay presencia en los últimos dos años.

Gráfica 1. Comparativa de porcentajes de temáticas principales por décadas

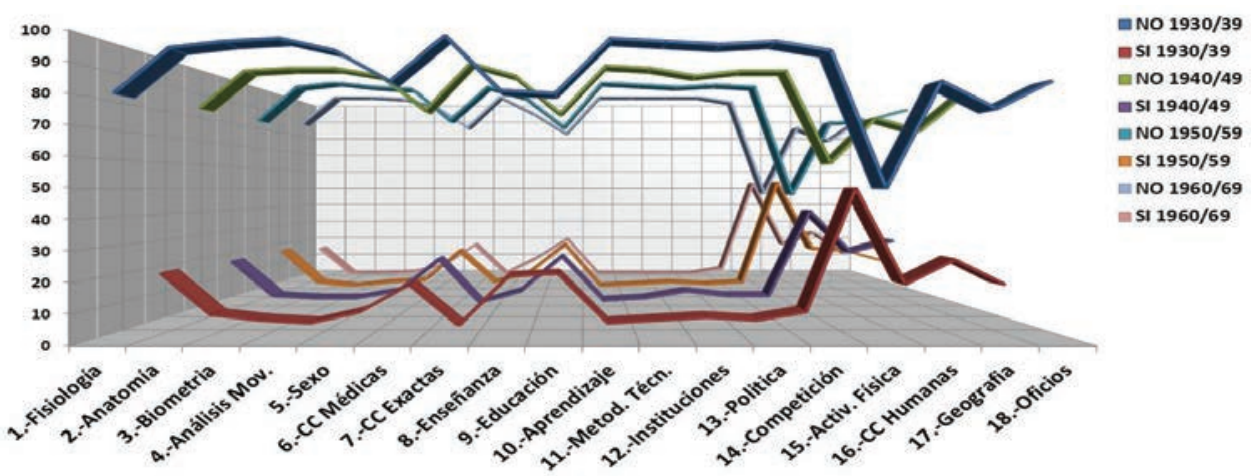


Seguidamente, entramos en el grupo de categorías que hemos encuadrado dentro de las temáticas relacionadas con la "Formación". En la Gráfica 1 podemos ver con detalle los porcentajes de las categorías: enseñanza, educación, aprendizaje y metodologías técnicas. Centrándonos en la "Enseñanza", en la primera década se publicaron 15,95\% de escritos, lo que corresponde a 26 de los 167 artículos publicados en esos años. Es la segunda década la que menos documentos presenta (4\%). La diferencia entre décadas fue significativa con un valor $<0.001$. En relación a la categoría "Educación" se publicaron el $21 \%$ de los 842 escritos totales. Este porcentaje se divide en: $18 \%$ en los primeros diez años, $20 \%$ en la segunda década, $23 \%$ en la tercera y $21 \%$ en los últimos años. Si atendemos ahora a la categoría "Aprendizaje" se puede observar que sólo se publicaron trabajos hasta el año 1949, no encontrándose ninguno más para los siguientes trece años. Durante las dos décadas en las que sí aparecen escritos, se hace con un $1 \%$ de las publicaciones totales, lo que corresponde a 5 artículos del total. Por último, si atendemos la categoría "Metodologías técnicas", la presencia de escritos durante las cuatro décadas no fue superior al $2 \%$, publicándose tres, cinco, seis y un escrito en cada una de las décadas.

Bajo una mirada global, la presencia de estas cuatro categorías, pertenecientes a la orientación temática "Formación", es variable, sobre todo en la categoría "Educación", donde la mayor recurrencia corresponde a formación profesional y formación continua. Situación diferente presentan los escritos que abordan los sistemas educativos, donde se explicita la subcategoría educación universitaria, que comienza a aparecer a partir de 1940. El tema de los recursos materiales se referencia, únicamente, en la segunda y tercera década, y el tema de los actores escolares muestra presencia entre la primera y tercera década, aunque en porcentajes muy bajos. Sobre orientación escolar siempre aparece algún escrito y, por último, en la subcategoría programas de estudio, sólo aparecen desde la segunda década.

Dentro de la temática "Organización”, en la Gráfica 1 podemos ver representadas las categorías "Instituciones", "Política” y "Competición”. En primer lugar, se observa que en la segunda década fue en la que más publicaciones se realizaron sobre las instituciones (9\%). La diferencia entre décadas para esta categoría fue significativa (p-valor 0.005), lo que se explica porque los escritos durante la tercera década tienen mucha mayor presencia que en las otras tres. Respecto a la categoría política, ésta tiene mayor presencia en los artículos correspondientes a la segunda década (3\%), manteniendo en las otras décadas valores porcentuales similares. Por último, la categoría competición tiene una mayor presencia en la primera década (7\%), seguida de la segunda y última década con un $4 \%$. El porcentaje para la segunda fue de un $2 \%$ del total.

Desde una mirada global, podemos observar que la temática "Organización" que acoge las categorías anteriormente analizadas, tiene una presencia constante durante las cuatro décadas, salvo algunas excepciones, como es el caso de los escritos relacionados con la descripción de instituciones deportivas, que tuvieron presencia (aunque no muy alta) desde la segunda década, y los artículos que referenciaban a instituciones nacionales, que fue perdiendo presencia a partir de la tercera década, especialmente en relación con las temáticas vinculadas con el ejército, que dicho sea de paso es muy poco referenciado en términos estadísticos. Por último, importa mencionar que las instituciones del estado y las colectividades referenciadas no tienen presencia en la última década.

Respecto a las temáticas relacionadas con la motricidad, identificadas dentro de la categoría "Actividad física", podemos ver en la Gráfica 1, en primer lugar, que es la que 
mayor presencia tiene en el análisis realizado. Su presencia se sitúa en torno al valor del $50 \%$ en tres de las cuatro décadas estudiadas (primera, tercera y cuarta). Además, debemos tener en cuenta que en la segunda década también presenta un valor alto, cercano al $40 \%$. Entrando en un análisis más minucioso de las subcategorías que la integran, podemos ver que los artículos relacionados con gimnasia médica y gimnasia deportiva prácticamente desaparecen en la última década. Ocurre algo similar para los deportes de invierno y los deportes en la naturaleza. El deporte universitario comienza a tener presencia a partir de la tercera década, y las actividades recreativas desde la segunda década. Las demás subcategorías se presentaron a lo largo de las cuatro décadas de estudio.

En cuanto al análisis de las categorías "Ciencias-Humanidades" y "Geografía" (Gráfica 1), podemos ver que en el caso de Ciencias-Humanidades, la década con mayor porcentaje de publicaciones (23\%) es la segunda, seguida de la tercera y la cuarta $(17 \%$ respectivamente), mientras que la primera década tiene un $12 \%$. Si entramos en el análisis de las subcategorías que la integran, podemos observar una cierta constancia en su presencia, a excepción de los escritos relacionados con "Educación popular", presentes sólo en la segunda y tercera década, "Edades-Adolescentes" en la primera y tercera década; los temas de "Psicología" aparecen a partir de la segunda década, y los temas de "Filosofía" desaparecen en la última década. Si detenemos la mirada en la categoría "Geografía", podemos observar que el $27,5 \%$ de las publicaciones que se realizaron en la segunda década descendieron a un $24 \%$ en la primera y la cuarta, quedando la tercera por debajo del $20 \%$. Las diferencias entre el número de publicaciones respecto a las décadas fueron significativas para esta temática (mayor porcentaje de publicaciones en la tercera década con respecto a las demás), con un p-valor 0.033. La única subcategoría ausente es "Otros", que sólo aparece, mínimamente, en la última década.

Para finalizar este análisis, podemos ver en la Gráfica 1 los porcentajes de presencia de la categoría "Oficios y funciones" en cada una de las décadas estudiadas. Ésta presenta valores poco significativos, apareciendo su máximo en la primera década, con un $11 \%$ de los escritos, que supone a su vez el $14 \%$ del total publicado en la década. Respecto al resto de las décadas, sus valores se sitúan en torno al $10 \%$.

Respecto al análisis bivariante realizado para ver las relaciones existentes entre las temáticas y su presencia en cada una de las décadas, podemos observar que éste muestra un recorrido temporal parcelado de la presencia de publicaciones, considerando las temáticas más y menos desarrolladas. Las consideraciones más significativas a tener en cuenta son las siguientes:

- Primera década: las categorías más representadas con diferencias significativas son, por orden de mayor a menor, "Actividad física" (EF utilitaria y profesional ${ }^{4}$ ), "Geografía" (Europa) y "Educación" (formación continua).

- Segunda década: "Actividad física" (EF utilitaria y profesional) y "Geografía” (América Latina) que mantiene su posición respecto a la década anterior, con la salvedad de una mayor presencia de América Latina respecto a Europa. El cambio más significativo se produce con "Ciencias-Humanidades" (sociología-educación popular), que sustituye en el tercer lugar a "Educación".

Los subtemas colocados a continuación son los que se llevan el mayor porcentaje de esta categoría mayormente representada por década. 
- Tercera década: debemos tener en cuenta que es el periodo donde existió un mayor número de publicaciones en total (341). "Actividad física" mantiene su primacía, pero es significativo reseñar que las categorías "Educación" (sistema educativo universitario) y "Ciencias médicas" (medicina deportiva y salud) sustituyen a "Geografía” y "Ciencias-Humanidades" respecto a la década anterior.

- Cuarta década: debemos tener presente que es la que muestra un rango menor de años ${ }^{5}$. En ella nuevamente "Actividad física" ocupa el primer lugar, seguida de "Geografía" (América Latina y Europa), y "Educación” (sistema educativo universitario).

Sintetizando lo expuesto, podemos destacar que la categoría "Actividad física" es la más potente en las cuatro décadas, seguida de "Geografía" y "Educación", que aparecen entre las tres primeras en tres de las cuatro décadas. Por último, es reseñable la presencia de "Ciencias-Humanidades" y "Ciencias médicas", que adquieren presencia en la segunda y tercera década, respectivamente.

Para cerrar este análisis de resultados, y en relación al análisis Chi cuadrado de significancia entre las variables, se ha detectado que cuatro de las 18 categorías poseen dicha significancia (Anatomía, Enseñanza, Ciencias-Humanidades y Geografía), es decir, presentan diferencias marcadas entre las cuatro décadas, lo que las convertiría en temáticas propias de cada periodo. Bajo este mismo análisis de Chi cuadrado, y considerando las orientaciones temáticas, se observa que no existe real significancia estadística de diferenciación entre las décadas para las temáticas en general, salvo para la orientación "Cuerpo biológico" que disminuye su presencia en la tercera década, lo mismo que para el caso de "Humanidades". Sin embargo, este último dato estadístico también puede interpretarse por un alza significativa de número de artículos, lo que dio paso a la publicación de una mayor variabilidad de temáticas y, por consiguiente, una redistribución porcentual de la presencia de las mismas.

\section{CONSIDERACIONES FINALES}

Reconociendo que en la primera mitad del siglo XX la historia de la educación chilena nos presenta una educación nacionalista, utilitaria y orientada al desarrollo económico, podemos observar, al tenor de los resultados presentados, que la EF de la época ayudó y fue promotora de este modelo, desde la formación en los valores morales predominantes, y buscando, sobre todo, poner a la EF al servicio del logro de cuerpos saludables e higiénicos, dignos de una raza apta para la producción. Esta realidad queda en evidencia desde las temáticas que los intelectuales de la época plasmaron en sus escritos en este segundo tercio del siglo XX. En este sentido, la Revista Chilena de EF se orientó, principalmente, a una EF utilitaria y profesional, donde las experiencias europeas de la década de los treinta fueron su gran referente teórico. El mejoramiento de la raza era el objetivo y para ello la EF se apoyaría en las ciencias médicas, afirmación que podemos corroborar a partir de la concentración de artículos relacionados con la temática en la década de los cincuenta.

La cuarta década consta de las publicaciones realizadas entre 1960 y 1962, debido al rango final elegido para esta investigación, y sobre la cual se sustenta el análisis y la relevancia de los escritos de estos profesionales e intelectuales, cuando en Chile sólo existía una institución formadora de profesionales de la Educación Física. 
Como es posible ver, el actual discurso pedagógico de la EF orientado a la salud corporal no es una innovación que surge de los nuevos dictámenes de la política educativa para el siglo XXI, sino más bien, una constante histórica del rol de la EF en Chile. Ahora bien, si observamos los datos que arroja, en tres ocasiones, el Sistema de Medición de la Calidad de la EF (SIMCE) en Chile, podemos notar en ella la fuerte argumentación de los objetivos de esta asignatura curricular en base a los parámetros de la condición física con fines biomédicos. El discurso de los alarmantes signos de obesidad, sobrepeso y sedentarismo de los niños, y sobre todo de las niñas en edad escolar, sumado a las investigaciones del Instituto de Nutrición y Tecnología en Alimentos (INTA), curiosamente organismo de la Universidad de Chile, se han tornado en el sustento de programas gubernamentales como "Elige Vivir Sano".

Es significativo comprobar cómo el debate de las metodologías, contextos y objetivos de la EF han sido relegados a mínimos cuestionamientos, al igual como ha ocurrido a lo largo de las cuatro décadas analizadas de la revista, donde los escritos relacionados con metodologías y aprendizaje arrojaron mínimas presencias (menos del 2\%). Como hemos podido comprobar, la preocupación por la formación y el perfeccionamiento docente de EF se ha centrado en la operativización de los mecanismos de salubridad poblacional gubernamental, no dejando espacio a cuestionamientos más profundos como el objeto y el rol de la EF para la trasformación social o la superación de las desigualdades sociales.

Por último, el análisis realizado evidencia la presencia de múltiples temáticas, aunque debemos tener en cuenta que la mayor concentración de artículos se produce en torno a los trabajos relacionados con la EF utilitaria y profesional, dando con esto interesantes antecedentes históricos para futuras investigaciones relacionadas con la forma en la que se concibió el ideario del profesional de la disciplina, sobre todo al iniciar su labor de difusión (década de los treinta), donde se concentró una alta producción de escritos relacionados con el perfeccionamiento docente y, posteriormente, una mayor concentración hacia la formación universitaria, todo en función de los sistemas gimnásticos y sistemas deportivos extranjeros.

Además, el análisis realizado nos permite decir que el origen histórico de la disciplina educativo-física está fuertemente argumentado por parámetros europeos. Sin embargo, sería interesante corroborar estos hechos históricos analizando en profundidad los escritos cuyo aumento durante la década del cuarenta referenció la realidad latinoamericana de la EF, lo que también pudiese dar luces del instante en el que la EF en América Latina buscó validar su propia historia.

\section{REFERENCIAS BIBLIOGRÁFICAS}

Cornejo, M., Matus, C. y Vargas, C. (2011). La EF en Chile: Una aproximación histórica. Revista Digital Efdeportes, vol. 16, n. 161. Recuperado de http://www.efdeportes.com/efd161/laeducacion-fisica-en-chile.htm, consultado en marzo de 2013.

Flores, N. y Rivera, J. (2003). Historia de la educación chilena. Antofagasta: Ediciones Centro de Educación a Distancia de la Universidad Católica del Norte.

Galdames, L. (1912). Educación económica e intelectual. Santiago de Chile: Imprenta Universitaria.

Godoy, P. (2007). Nacionalidad y Educación. Santiago de Chile: Ediciones Nuestramérica.

Gutiérrez, S. (1933). Los institutos de Educación Física y su organización. Tirada aparte de los 
Anales de la Universidad de Chile. Santiago de Chile: Prensa de la Universidad de Chile.

Hevia, P., Fernández, J. y Home, D. (2010). Una experiencia educativa: Sociedad de instrucción primaria. 150 años. Santiago de Chile: Origo.

Instituto de Educación Física. (1936). Joaquín Cabezas García. Sus Bodas de Oro con la enseñanza. 1886-1936. Santiago de Chile: Imprenta Universitaria. (1934). Notas editoriales. Boletín de Educación Física, vol. 1, n. 1, s/p.

Labarca, A. (1939). Historia de la enseñanza en Chile. Santiago de Chile: Publicaciones de la Universidad de Chile.

Martínez, F. (2012). Prácticas y discursos: La formación de la Educación Física en Chile. 18891920. Revista Digital Efdeporte, vol. 17, n. 171. Recuperado de http://www.efdeportes.com/efd171/ la-educacion-fisica-en-chile-1889-1920.htm, consultado en marzo de 2013.

Moulian, T. (2006). Fracturas: De Pedro Aguirre Cerda a Salvador Allende (1938-1973). Santiago de Chile: LOM Ediciones.

Muñoz, C. (2001). Bases para abordar el origen y evolución de las Políticas Estatales chilenas en Educación Física en el período 1889-1929. Plataforma Universidad de Chile. Recuperado de http:// www.lapetus.uchile.cl/lapetus/search.php?key=deporte, consultado en marzo de 2013.

Remans, A. (2000). Información y documentación deportiva en Europa. Revista General de Información y Documentación, vol. 10, n. 1, 17-29.

Ruiz, C. (2010). De la república al mercado: Ideas educacionales y políticas en Chile. Santiago de Chile: LOM Ediciones.

Salas, J. A. (2009). Génesis y difusión de la Educación Física en Cuba (1800-1901). Tesis de Doctorado, Departamento de Historia y Teoría de la Educación, Universidad de Salamanca, España. Recuperado de http://gredos.usal.es/jspui/bitstream/10366/76296/1/DTHE_Salas_Rondon_JA_ Genesis_y_difusion.pdf, consultado en julio de 2013.

Toro, S. (2005). Una aproximación epistemológica a la didáctica de la motricidad desde el discurso y la práctica docente. Tesis de Doctorado, Pontificia Universidad Católica de Chile, Santiago de Chile. Recuperado de http://www.kon-traste.com/pdf/temasinvestigadores/TESIS_DOCTORAL_ SERGIO_TORO_DE_CHILE.pdf, consultado en agosto de 2013. 